\title{
The Development of Hydrophobic and Superhydrophobic Cementitious Composites
}

\author{
S. W. Muzenski, I. Flores-Vivian, and K. Sobolev \\ Department of Civil \& Environmental Engineering, University of Wisconsin-Milwaukee
}

\begin{abstract}
Freezing and thawing of water in saturated concrete induces stresses, cracks, spalling, and eventually allows chlorides, sulphates and other ions to penetrate through the porous space of concrete causing the corrosion and ultimately structural failure. These detrimental factors are limited if concrete has the ability to repel water. Water repellant concrete was achieved through internal and external surface applications. Hydrophobicity, over- and super-hydrophobicity are demonstrated by the ability of a surface to repel water and are characterized by contact angles. In cementitious composites internal hydrophobization can be achieved through the addition of superhydrophobic admixtures to fresh concrete resulting in an air void system with small, well-dispersed air bubbles to provide superior resistance to freezing and thawing. Hydrophobic, over- and super-hydrophobic surface coatings were achieved by the design of hierarchical surfaces tailoring the fiber content, mixture proportion, and superhydrophobic emulsions. Contact angle tests were used to characterize the developed coatings. The use of internal hydrophobization improves freezing and thawing resistance of fiber-reinforced composites as demonstrated by a durability factor of 100 through as many as 700 accelerated $\left(-50^{\circ} \mathrm{C}\right.$ to $\left.20^{\circ} \mathrm{C}\right)$ cycles in $5 \% \mathrm{NaCl}$ solution.
\end{abstract}

Keywords: contact angles, freeze-thaw resistance, hierarchical surfaces, hydrophobicity, superhydrophobicity.

\section{INTRODUCTION}

The hydrophobicity of a material is defined as the ability to repel water and depends on the surface chemical composition and the surface geometry (micro- and nano-structural morphology) (Liu, 2006). The contact angle between a drop of water and the surface is generally used as an indicator of hydrophobicity or wetability. When the contact angle is greater than $90^{\circ}$, it indicates hydrophobicity, while a contact angle less than $30^{\circ}$ denotes hydrophilicity, which is the tendency of a surface to become wet or to absorb water, as shown in Figure 1. Common concrete is an example of a hydrophilic mesoporous material which absorbs water. The superhydrophobicity corresponds to contact angle between $150^{\circ}$ and surfaces with intermediate properties (with high contact angles between $120^{\circ}$ and $150^{\circ}$ ) above typical values for hydrophobic materials, are called "overhydrophobic." The water contact angle with a solid surface can be measured by goniometer or tensiometer (Nosonovsky, 2007).

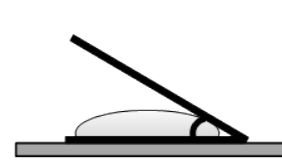

Hyrdophilic surface:

$\Theta<30^{\circ}$

(normal concrete)

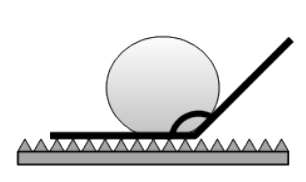

Overhydrophobic surface:

$120^{\circ}<\Theta<150^{\circ}$

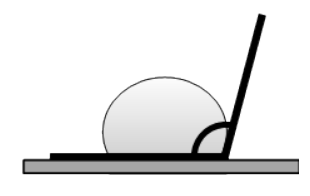

$90^{\circ}<\Theta<120^{\circ}$

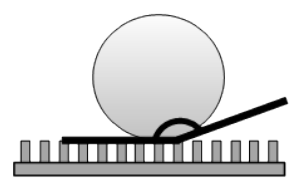

Superhydrophobic surface: $\Theta>150^{\circ}$
Hydrophobic surface:

Figure 1. Hydrophillic, hydrophobic, overhydrophobic, and superhydrophobic surfaces (Flores-Vivian et al., 2013).

Superhydrophobic surfaces with hierarchical roughness patterns imposed over larger roughness patterns have generated interest due to their potential in industrial applications (mainly, for selfcleaning). These surfaces mimic the lotus leaf surface, which is well known for its superhydrophobicity and self-cleaning properties, 
(lotus-effect). Mimicking living nature for engineering applications is called "biomimetics," and biomimetic approaches can be used to synthesize hydrophobic and superhydrophobic concrete (Sobolev \& FerradaGutiérrez, 2005; Sobolev \& Batrakov, 2007; Poole, n.d.; Nosonovsky, 2011; Nosonovsky \& Bhushan, 2009; Hejazi et al., 2013). Waterproofing and the incorporation of hydrophobic additives into the concrete matrix are two approaches used to improve the physical properties of concrete. The first approach consists of using hydrophobic materials on the surface of concrete to repel water (Moriconi \& Tittareli, 2009), which also improves the freeze thaw durability of concrete. The type of material and quantity used affects its concrete protecting efficiency (Raupach \& Wolff, 2005). The second approach consists on creating a hydrophobic concrete or cement matrix using admixtures (Sobolev \& Batrakov, 2007; Popovics, 1982; Hekal \& Abd-El-Khalek, 1999, 2000). The polymethylhydrosiloxane (PMHS) based admixtures react with cement based systems to release hydrogen and create small $(10-100 \mu \mathrm{m})$, well dispersed air voids throughout the cementitious matrix. The walls of these voids are then coated with sub-micro and nano-sized particles to produce the hierarchical surface roughness to achieve superhydrophobic properties (Figure 2). The addition of a hydrophobic admixture into the concrete mix represents a viable possibility to achieve good quality concrete and may prove to be good alternative to conventional air entraining admixtures. These admixtures can tailored to result in air voids of optimal size and spacing to provide superior freezing and thawing resistance while at the same time resulting in little to no reduction in compressive strength (Sobolev et al., 2010).

\section{EXPERIMENTAL INVESTIGATION}

\subsection{Materials}

Fiber reinforced mortar specimens used for freezing and thawing tests were prepared using a Type I portland cement (PC), ground granulated blast furnace slag (GGBFS) both from Lafarge, and ASTM C778 standard-graded silica sand. Polyvinyl alcohol (PVA) fibers (RECS $15 \times 12 \mathrm{~mm}$ Kuralon K-II) with a diameter of $15 \mathrm{dtex}(0.04 \mathrm{~mm})$ and length of $12 \mathrm{~mm}$ were used in this study. These fibers had a Young's modulus of $40 \mathrm{GPa}$ and a tensile strength of 1.6 $\mathrm{GPa}$. The high-range water-reducing admixture used in the study was commercially available polycarboxylate ether superplasticizer (PCE/SP) with a $31 \%$ solid concentration.
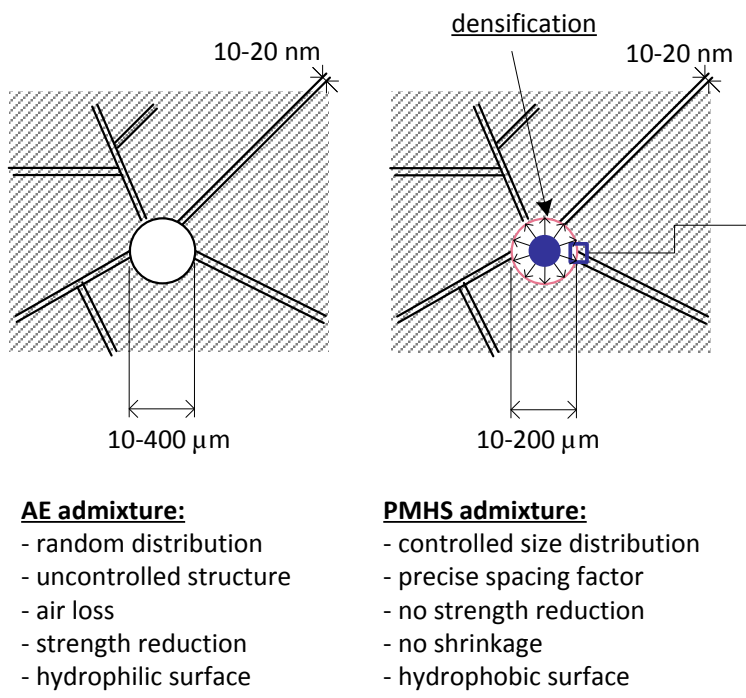

PMHS admixture:

- controlled size distribution

- precise spacing factor

- no strength reduction

- no shrinkage

- hydrophobic surface

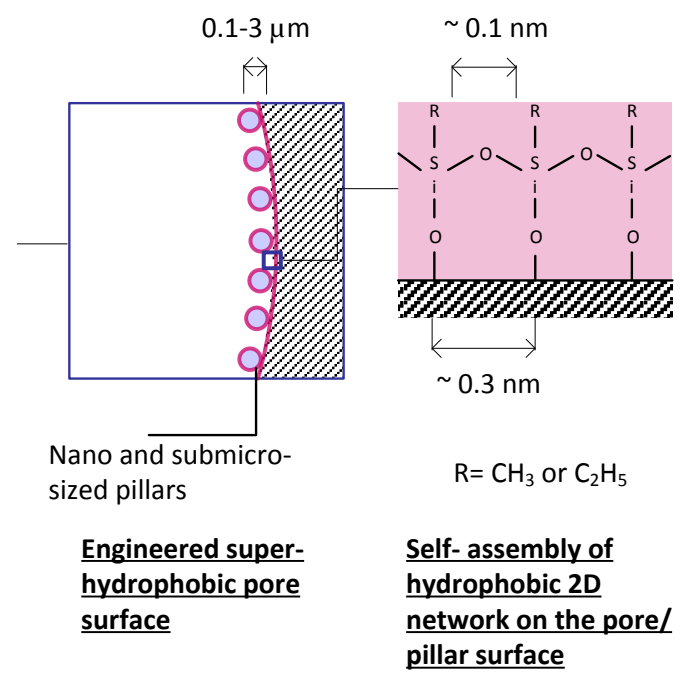

Figure 2. How the superhydrophobic hybridization of concrete works (Sobolev et al., 2010).

Water soluble polyvinyl alcohol (PVA) was selected for emulsion stabilization because of its nonionic properties and perfect compatibility with concrete materials (Kim \& Robertson, 1999). A hydrolyzed (98\%) PVA with molecular weight of 16,000 from Acros Organics was used to reduce the tendency of foam formation. Deionized (DI) water was used as the dispersion medium for the emulsions. Polymethyl-hydrogen siloxane (PMHS) oil (Xiameter ${ }^{\circledR}$ MHX-1107) from Dow Corning with a specific gravity of 0.997 (at $25^{\circ} \mathrm{C}$ ) and a viscosity of $30 \mathrm{cSt}$ was used as the hydrophobic agent. This product contains $85-100 \%$ of polymethylhydrogen siloxane as an active ingredient. $\mathrm{Nano}^{-\mathrm{SiO}_{2}}$ (NS) from MKNANO with a purity of $99.5 \%$, average particle size of $15 \mathrm{~nm}$, and a Brunauer-EmmettTeller (BET) surface area of $112 \mathrm{~m}^{2} / \mathrm{g}$ was used. 
Silica fume (SF) from Elkem and metakaolin (MK) from Burgess Optipozz were used as the sub-micro particles in the emulsion. The morphology of MK and SF were analyzed by the scanning electron microscope (SEM) technique. Rough and flaky particles with sizes from 0.8 to $12 \mu \mathrm{m}$ with a certain degree of agglomeration were found in the metakaolin. Spherical particles with sizes from 0.2 to $1 \mu \mathrm{m}$ with a certain degree of agglomeration were found in silica fume.

\subsection{Methods}

To prepare the emulsions, water was used as a dispersion medium, water-soluble PVA as surfactant and PMHS as the dispersion phase. Micro-particles of silica fume or metakaolin were used to stabilize (Binks \& Lumsdon, 2000; Aveyard et al., 2003; Ngai et al., 2006) and modify the emulsion using two different approaches: "shell" and "core" as described in CFIRE 07-03 (Sobolev et al., 2013) and explained in detail in Flores-Vivian et al. (2013). For external applications of superhydrophobic emulsions $\left(E_{1 s}\right.$ and $\left.E_{1 S R}\right)$, the concentrations of surfactant, siloxane, and silica fume or metakaolin were $3.485,25$, and $5 \%$ by the weight of the emulsion respectively for $E_{1 S}$ and emulsion $E_{1 S R}$ was produced by diluting 1 part of $E_{1 s}$ emulsion with 4 parts of DI water (Table 1). For the internal hydrophobization $\left(E_{2}\right)$, the concentrations of surfactant, siloxane, metakaolin, and nano- $\mathrm{SiO}_{2}$ were kept constant at 3.50, 25, 0.4, and $0.1 \%$ by the weight of the emulsion respectively.

Table 1. Emulsion proportions.

\begin{tabular}{l|cccccccc}
\hline $\begin{array}{c}\text { MIXTURE } \\
\text { ID }\end{array}$ & $\begin{array}{c}\text { DI } \\
\text { Water } \\
\%\end{array}$ & $\begin{array}{c}\text { PVA } \\
\%\end{array}$ & $\begin{array}{c}\text { PMHS } \\
\%\end{array}$ & $\begin{array}{c}\text { SF } \\
\%\end{array}$ & $\begin{array}{c}\text { Biocide } \\
\%\end{array}$ & $\begin{array}{c}\text { MK } \\
\%\end{array}$ & $\begin{array}{c}\text { NS } \\
\%\end{array}$ & $\begin{array}{c}\text { Total } \\
\%\end{array}$ \\
\hline E1SShell & 66.2 & 3.5 & 25 & 5.0 & 0.30 & 0.0 & 0.0 & 100 \\
E1ShellR & 93.2 & 0.7 & 5 & 1.0 & 0.06 & 0.0 & 0.0 & 100 \\
E2Core & 71.0 & 3.5 & 25 & 0.0 & 0.00 & 0.4 & 0.1 & 100 \\
\hline
\end{tabular}

Mortars for application of external hydrophobic coatings were produced with varying W/C, S/C and high range water reducing admixtures (Table 2). The PVA fiber volume was held constant at $1 \%$ for all mixes. These mortars were produced in accordance with ASTM C305 and placed in tile molds of $15 \times 15$ $\times 8 \mathrm{~mm}$. The samples were hardened in molds for 24 hours at $23 \pm 3^{\circ} \mathrm{C}$ and at least $90 \%$ of relative humidity. Specimens were demolded after 24 hours and cured in tap water for 72 hours, then dried for 36 hours at $60-70^{\circ} \mathrm{C}$.

To investigate the contact angle of water on the mortar tiles, the tiles were roughened with silicon carbide grinding paper with a grit of 60 for 30 seconds in order to expose the fresh surface and sand aggregates. The tiles were then placed in an ultrasound to remove any loose particles from the surface. The samples were then placed in an oven at $40^{\circ} \mathrm{C}$ for 24 hours to remove any excess moisture and then were allowed to dry at room temperature for $48 \mathrm{hrs}$. The wetting properties of the tiles were examined by measuring the water contact angle using the Kruss DSA100 "Drop Shape Analysis System" goniometer by placing $20 \mu$ of water onto each tile surface using the instrument's automatic syringe.

Table 2. Mortar mixture proportions of tile for externa hydrophobic applications.

\begin{tabular}{ccccc}
\hline MIXTURE ID & W/C & S/C & $\begin{array}{c}\text { PCE/SP } \\
\text { (solid \% of cement) }\end{array}$ & $\begin{array}{c}\text { PVA Fibers } \\
\text { (\% Vol) }\end{array}$ \\
\hline M1 & 0.25 & 0.0 & 0.14 & 1.0 \\
M2 & 0.30 & 1.0 & 0.10 & 1.0 \\
M3 & 0.40 & 2.0 & 0.10 & 1.0 \\
M4 & 0.45 & 2.5 & 0.10 & 1.0 \\
M5 & 0.50 & 3.0 & 0.10 & 1.0 \\
\hline
\end{tabular}

The fiber reinforced material for freezing and thawing tests was prepared in a 19 liter mixer as follows. First, $75 \%$ of tap water at room temperature was added to the mixing bowl along with the PCE/SP. Next, standard-graded silica sand was added and mixed at a low speed (107 rpm) for 30 seconds. Half of the PVA fibers were then added and mixed for 30 seconds, followed by the remaining PVA fibers for another 30 seconds. Half of the cementitious materials were added next and mixed for one minute, followed by the remaining cementitious materials for another minute. Finally, the remaining water along with the PMHS admixture was added and mixed at low speed for one minute, followed by 30 seconds of mixing on medium speed (198 rpm) (Sobolev et al., 2010). A portion of ECC/SECC was then tested for flow, followed by mixing for 30 seconds at a medium speed before placing into the $40 \times 40 \times 160 \mathrm{~mm}$ beam molds for durability factor evaluation.

The samples were then covered with glass plates and placed in a curing room at standard temperature $\left(20 \pm 3^{\circ} \mathrm{C}\right)$ and a relative humidity of no less than 90\%, per ASTM C192. The samples were removed from the molds after 24 hours and placed in a lime water bath for an additional 13 days until the time of testing.

Freeze-thaw tests were performed in an accelerate manner (which is different from standard ASTM C666 procedures) with temperatures oscillating between $-50^{\circ} \mathrm{C}$ and $20^{\circ} \mathrm{C}$ (95\% relative humidity). This accelerated testing may provide a cycle multiplier of 5, meaning 700 freezing and thawing cycles would be equivalent to 3500 cycles tested using standard methods (Sobolev \& Batrakov, 2007). Temperatures were held at the extreme 
points for just under 2 hours and the temperature change occurred rapidly (approximately $15-25$ minutes from minimum to maximum temperature and vice versa). This provided a single cycle with a total duration of 4 hours. Samples were tested in 5\% $\mathrm{NaCl}$ solution which was replaced every 50 or 100 cycles when the samples were tested for durability factor. The durability factor was calculated based on standard calculations of relative dynamic modulus of elasticity from ASTM C215.

Mixtures were prepared with $2.75 \%$ by volume PVA fibers. Samples were produced at a water to cementitious material ratio of both 0.30 and 0.45 with a sand to cementitious material ratio of 0.5 and 1.0 respectively. Each mixture was created with and without a single dose of emulsion ( $0.25 \mathrm{~g}$ of siloxane to 1 liter of entire composite mix). The experimental mixtures are summarized in Table 3.

Table 3. Experimental program for samples with internal hydrophobization.

\begin{tabular}{cccccc}
\hline $\begin{array}{c}\text { MIXTURE } \\
\text { ID }\end{array}$ & W/CM & S/CM & $\begin{array}{c}\text { GGBFS } \\
(\%)\end{array}$ & $\begin{array}{c}\text { Emulsion } \\
\text { Dosage } \\
(\mathrm{g} / \text { liter })\end{array}$ & $\begin{array}{c}\text { PCE/SP } \\
\text { Content } \\
(\%)\end{array}$ \\
\hline REF30 & 0.30 & 0.5 & 50 & 0 & 0.125 \\
REF45 & 0.45 & 1.0 & 50 & 0 & 0.050 \\
E30 & 0.30 & 0.5 & 50 & 0.25 & 0.125 \\
E45 & 0.45 & 1.0 & 50 & 0.25 & 0.050 \\
\hline
\end{tabular}

\section{RESULTS AND DISCUSSION}

The effects of fibers, the concentration of cementitious material, and the concentration of the hydrophobic agent (emulsion type) were investigated for contact angle. Emulsions $E_{1 s}$ and $E_{1 S R}$ with 25 and $5 \%$ of siloxane, respectively, were used for coating of the tiles (Figure 3). Due to the high water absorption, the contact angles for uncoated specimens were very small (0 to 20 degrees). Uncoated specimens absorb most of the water since portland cement based materials are hydrophilic. The combination of fibers, submicroand nano-sized particles of silica fume, and the hydrophobic agent increased the contact angles for all specimens which provide the micro/nanoroughness of a hierarchical structure required for superhydrophobicity effects. The diluted emulsion $\left(E_{1 S R}\right)$ demonstrated a reduced contact angle when the W/C and S/C was increased. The samples produced with high W/C ratio and S/C ratio had higher porosity and thus the hydrophobic agent may be absorbed by the porous network, which may be the reason for the lower contact angle seen in samples $E_{1 S R \_} M 4$ and $E_{1 S R \_} M 5$. Non diluted emulsion $\left(E_{1 S}\right)$ produce a thicker coating therefore eliminating the effects on contact angle due to the surface roughness of the tile itself when lower W/C ratio and S/C ratio is considered. The surface roughness of higher W/C and S/C samples were still able to contributed to the contact angle for this nondiluted samples which why there is an incremental increase in contact angle for $E_{1 s}$ emulsions.

For the specimens coated with $E_{1 S}$, the contact angle increased with the reduction of the cementitious materials and sand content. The roughness introduced by the aggregates increased the hydrophobicity of tiles coated by $E_{1 s}$. On thicker coats $\left(E_{1 S}\right)$, the higher roughness induced by the aggregates had a positive effect on the hydrophobicity of the material. The higher concentration of hydrophobic agent in $\mathrm{E}_{1 \mathrm{~s}}$ seemed to minimize the roughness effect of the fibers. The diluted emulsion $E_{1 S R}$ developed a thin coat, resulting in similar results as the contact angle where some hydrophobic agents were absorbed in the porous samples ( $E_{1 S R} M 4$ and $\left.E_{1 S R \_} M 5\right)$.

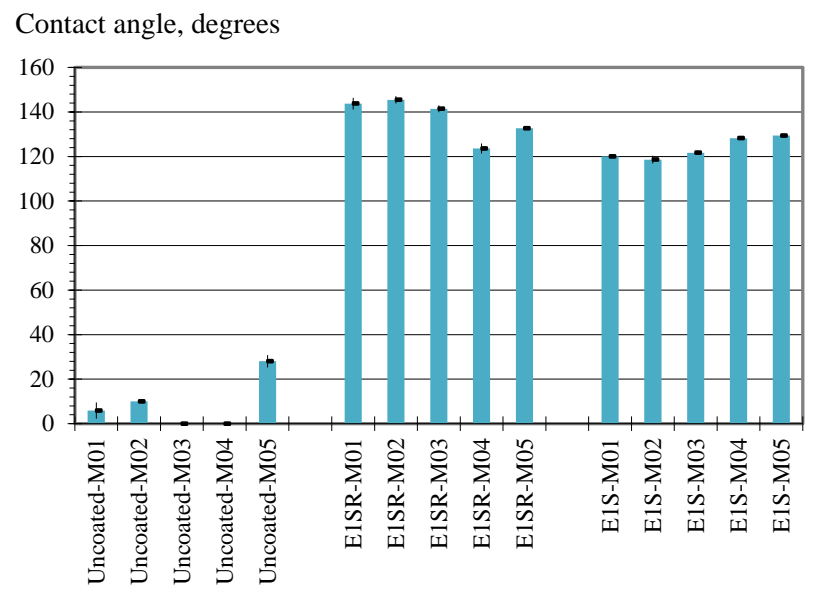

Figure 3. Contact angle for tiles coated in $E_{1 S}$ and $E_{1 S R}$.

Results from the freeze-thaw testing demonstrate that the main factor affecting the durability of the material is the W/CM ratio (Figure 4). All samples with a lower W/CM ratio displayed exceptional performance through as many as 700 cycles by maintaining of durability factor of 100 or more, meaning that the dynamic modulus of elasticity is the same if not higher through cycling compared to untested specimens. For these samples an increase in durability factor can be seen through the first 200 cycles. This can be attributed to the continued strength gain (since the samples were placed in the chamber at 14 days). This, however, was not the case for higher W/CM ratio samples, demonstrating that the weaker matrix with larger capillary porosity was subjected to immediate 
deterioration due to severe exposure. The samples that included emulsions (E30 and E45) demonstrated superior performance than the same samples without the emulsion. The increase in performance was significant for the samples with higher W/CM however, displayed only a slight increase for samples with a lower W/CM. It may be assumed that if tested further, deterioration of lower W/CM samples will occur, in this case, the superhydrophobic admixtures may demonstrate significant improvement. This can be seen as the deteriorated samples with a higher W/CM ratio and superhydrophobic admixtures (E45 Salt) displayed better performance compared to the samples without (REF45 Salt).

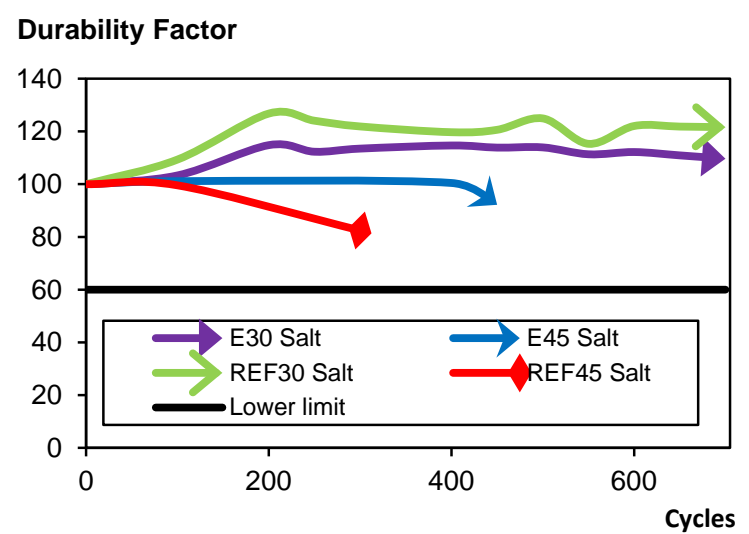

Figure 4. Durability factor for samples during freezing thawing in $5 \% \mathrm{NaCl}$ solution.

\section{CONCLUSION}

The application of hydrophobic emulsions as a coating on mortar tiles proved to result in high contact angles, especially when diluted emulsions were considered. Samples with a stronger cementitious matrix (low W/C and low S/C) demonstrated superior performance as well. This could be attributed to the fact that when highly porous samples are used in combination with diluted emulsions, the emulsions become absorbed in the tiles and thus reduce hydrophobicity effects. The opposite effect occurs when undiluted emulsions are used in combination with rougher tile surfaces (high W/C and high S/C) as the excess hydrophobic agents produce a thicker coat and eliminate the effects of hydrophobicity from the surface roughness of the tile itself.

Based on the durability factor results, it is clear that a lower W/CM provides a better freeze-thaw response. Additionally, the use of superhydrophobic admixtures even further improves the behavior in salt water solutions. This is evident as the these samples have lower permeability and absorption due to their superhydrophobic air voids and thus better freezing and thawing resistance.

Based on these results, it is clear that a low W/C improves hydrophobic properties of surface coatings and also drastically improves the freezing and thawing resistance. When adding superhydrophobic emulsions internally to low W/C samples, the freezing and thawing resistance is even further improved. The combination of these two techniques should result in an ultra-durable cementitious material. Further research may need to be performed to demonstrate this hypothesis. Further research may also be required to improve the hydrophobicity of emulsions to achieve contact angles of more than $150^{\circ}$ to be considered superhydrophobic.

\section{ACKNOWLEDGEMENTS}

The authors thank the National Center for Freight \& Infrastructure Research \& Education for funding this project through grants CFIRE 04-09, CFIRE 05-10, CFIRE 06-03, and CFIRE 07-03. Support from the University of Wisconsin-Milwaukee Research Growth Initiative and the National Science Foundation is acknowledged. The authors also thank Kuraray, Nycon, Lafarge, and Handy Chemicals for donation of materials used in this study.

\section{REFERENCES}

Aveyard, R., Binks, B., \& Clint, J. (2003). Emulsions stabilised solely by colloidal particles. Advances in Colloid and Interface Science, 100-102(28), 503-546.

Binks, B., \& Lumsdon, S. (2000). Influence of particle wettability on the type and stability of surfactant-free emulsions. Langmuir, 16(23), 8622-8631.

Flores-Vivian, I., Hejazi, V., Kozhukhova, M. I., Nosonovsky, M., \& Sobolev,K. (2013). Selfassembling particle-siloxane coatings for transferring concrete towards superhydrophobicity. ACS Applied Materials \& Interfaces, 5(24), 13284-13294.

Hejazi, V., Sobolev, K., \& Nosonovsky, M. (2013). From superhydrophobicity to icephobicity: Forces and interaction analysis. Scientific Reports, 3. 2194.

Hekal, E. E., \& Abd-El-Khalek, M. (1999). Mechanical and physico-chemical properties of hardened portland cement pastes containing hydrophobic admixtures part 1: Compressive strength and hydration kinetics. ZKG International, 52(12), 697-700. 
Hekal, E. E., \& Abd-El-Khalek, M. (2000). Mechanical and physico-chemical properties of hardened portland cement pastes containing hydrophobic admixtures part 2: Physical properties and micro-structure. ZKG International, 53(3), 152-158.

Kim, J. H., \& Robertson, R. E. (1999). Structure and properties of poly(vinyl alcohol)-modified mortar and concrete. Cement and Concrete Research, 29(3), 407-415.

Liu, Y. (2006). Super hydrophobic surfaces from a simple coating method: A bionic nanoengineering apporach. Nanotechnology, 17, 3259-3263.

Moriconi, G., \& Tittareli, F. (2009). Effectiveness of surface or bulk hydrophobic treatments in cementitious materials. Protection of Historical Buildings, PROHITECH, 9, 1071-1075.

Ngai, T., Auweter, H., \& Behrens, S. (2006). Environmental responsiveness of microgel particles and particle-stabilized emulsions. Macromolecules, 39(23), 8171-8177.

Nosonovsky, M. (2007). Multiscale roughness and stability of superhydrophobic biomimetic interfaces. Langmuir, 23(6), 3157-3161.

Nosonovsky, M. (2011). Slippery when wetted. Nature, 477, 412-413.

Nosonovsky, M., \& Bhushan, B. (2009). Superhydrophobic surfaces and emerging applications: Non-adhesion, energy, green engineering. Current Opinions Colloid Interface Science, Volume 14, pp. 270-280.

Poole, B., n.d. Biomimetics: Borrowing from biology. Web. Retrieved from http://www.thenakedscientists.com/HTML/articles /article/biomimeticsborrowingfrombiology/

Popovics, S. (1982). Fundamentals of portland cement concrete. John Wiley and Sons, Inc.

Raupach, M., \& Wolff, L. (2005). Investigations on long-term durability of hydrophobic treatment on concrete. Surface Coatings International PartB: Coatings Transactions, 99(2), 127-133.

Sobolev, K., \& Batrakov, V. (2007). The effect of a PEHSO on the durability of concrete with supplementary cementitious materials. ASCE Journal of Materials in Civil Engineering, 19(10), 809-819.

Sobolev, K., \& Ferrada-Gutiérrez, M. (2005). How nanotechnology can change the concrete world: Part 2. American Ceramic Society Bulletin, 11, 16-19.

Sobolev, K., Nosonovsky, M., Krupenkin, T., FloresVivian, I., Rao, S., Kozhukhova, M. . . Rivero, R. (2013). Anti-icing and de-icing superhydrophobic concrete to improve the safety on critical elements on roadway pavements. Publication No. CFIRE 07-03.
Sobolev, K., Oliva, M., Tabatabai, H., \& Zhao, J. (2010). Superhydrophobic engineered cementitious composites for highway bridge applications: Phase I. Publication No. FHWA CFIRE National Transportation Center Project 04-09. 